\title{
Predictors of correct angulations to perform invasive retrograde left ventriculography - defined by cardiac 256 multi-sliced computed tomography
}

\author{
Anger $T^{1 *}$, Wolff $A^{1}$, Pabst $\mathrm{P}^{1}$, Bauer $\mathrm{V}^{3}$, Mänz $\mathrm{C}^{2}$, Jorbenadze $\mathrm{R}^{4}$, Schneider $\mathrm{M}^{5}$ and Oberhoff $\mathrm{M}^{1}$ \\ ${ }^{1}$ Department of Internal Medicine/Cardiology, Klinikum Calw-Nagold, Germany \\ ${ }^{2}$ Department of Radiology, Klinikum Calw-Nagold, Germany \\ ${ }^{3}$ Department of Internal Medicine/Cardiology, Klinikum Bayreuth, Germany \\ ${ }^{4}$ Department of Internal Medicine/Cardiology, University of Tübingen, Germany \\ ${ }^{5}$ Department of Internal Medicine/ Electrophysiology, Klinikum Sindelfingen, Germany
}

\begin{abstract}
Background: Correct angulation performing invasive retrograde probed left ventriculography in the cath-lab is crucial to define individual patient specific wall motion stress of the left ventricle. Since decades, left ventriculography is assessed in LAO $60^{\circ}$ and RAO $30^{\circ}$ - this can't be right. This study defines the correct angulations.

Methods: We used in total n=1000 cardiac 256 multi-sliced computed tomography 3D heart reconstructions retrospectively and reordered the heart in regard to the invasive left ventriculography angulations - thereby, we got the correct angulation parameters. We assessed additionally LV-hypertrophy, gender, age, body mass index (BMI), electrocardiogram (ECG) and QRS axis to characterize specific predictors.

Results: We got a statistically significant recipe of changed angulations: Older patients need LAO angle tipped to $50^{\circ}$, younger patients flatter to $42^{\circ}$. Male patients change the Caudal_LAO angle more to $20^{\circ}$, whereas female held the Caudal_LAO angle to $16^{\circ}$. In general, the ECG itself and pre-described LV-hypertrophy are without any impact. In regard to the QRS axis of the ECG only marked left axis deviation changes the angles: LAO 50\% Caudal_LAO 15 ${ }^{\circ}$, RAO 20\% Caudal_RAO $5^{\circ}$. LVhypertrophy changes Caudal_LAO more to $18^{\circ}$, BMI over 30 reduces Caudal_LAO to $11^{\circ}$. Only minor changes in the angulation Caudal_RAO: $4^{\circ}$ when BMI is more than 30 . No significant changes on RAO $30^{\circ}$.
\end{abstract}

Summary and Conclusion: We declare a recipe for correct angles performing invasive retrograde left ventriculography in the set-up of cath-lab: age-, LV hypertrophy, gender and BMI- dependent. Invasive cardiologists will be forced to follow the recipe of miscellaneous angulations while performing retrograde probed characterization of LV function.

\section{Introduction}

In general, left ventriculography is performed in the cath-lab through retrograde probing of the left ventricle (LV) with an obligate pigtail catheter (always $5 \mathrm{~F}$ thickness) and through invasive perfusion of $25 \mathrm{ml}$ contrast media in $10 \mathrm{ml} / \mathrm{sec}$ flooding in two angulations: RAO $30^{\circ}$ Caudal_RAO $0^{\circ}$ to characterize anterior, apical and inferior wall motion kinetics as well as the ejection fraction (EF [in \%]), and in $\mathrm{LAO} 60^{\circ}\left(90^{\circ}\right.$ to RAO $\left.30^{\circ}\right) /$ Caudal_LAO $0^{\circ}$ to characterize septal, posterior and lateral wall motion kinetics as well as to estimate left ventricular wall thickness in regard to define LV hypertrophy [1]. This procedure is done despite of several specific parameters of the patient like the height, the weight, the body mass index (BMI) respectively, LV-hypertrophy (result from transthoracic echocardiography), QRS axis of the electrocardiogram (ECG), gender, or age [2]. Known as epidemiological left turning of the heart in course of live, we are wondering about young patients in the cath-lab seen the left ventriculography done with same angulations as on old patients. It should be a more to the right emphasized heart in younger, a more to the left emphasized heart in older patients without further parameters impacting angulations performing left ventriculography. It is all over the world set to LAO $60^{\circ}$ and RAO $30^{\circ}$ without any changes and without any further angulations to Caudal - neither for LAO nor for RAO. In the literature, selected comparisons of LV function performed with left ventriculography in correlations to cardiac multi-sliced computed tomography or magnet resonance imaging are published without further impact to change angulations to correct angles assessing invasively LV characterization [3].

We decided to change and solve this issue addressing correct patient dependent individual angulations while performing invasive retrograde left ventriculography in the cath-lab. We defined correct angles, defined different groups of patients with specifically different angulations and therefore we defined specific predictors indicating the use of correct angles here in this study.

Approaching this effort, we collected in total $n=1000$ characterized left ventricles from patients from different study sides (multi-center:

${ }^{*}$ Correspondence to: Thomas Anger, Department for Internal Medicine, Kreisklinikum Calw-Nagold, Kliniken Calw, Germany, E-mail: th.anger@arcor.de

Key words: left ventriculography, angulations, cardiac computed tomography, predictors

Received: July 20, 2019; Accepted: July 26, 2019; Published: August 02, 2019 
Bayreuth, Sindelfingen, Tübingen, Calw) with performed cardiac 256 multi-sliced computed tomography under different indications (i.e. ruling out severe coronary artery disease, or to characterize the severeness of aortic valve stenosis) and we reordered 3-dimensionally in a single center manner (Calw) the heart to be able to characterize LV function as done in the invasive left ventriculography (Figure 1). The reordering figured out the correct angulations given through the CT program and we selected other parameters given through the patient characteristics (body mass index, LV-hypertrophy, QRS axis of the ECG, gender, and age).

We found several changes in the correct angulations dependent on abovementioned parameters and we defined predictors of correct use of angulations in the cath-lab while performing invasive retrograde left ventriculography in regard to characterize LV function.

\section{Methods}

\section{Study design and methods}

This is an observational blinded multi-center retrospective study of in total of 1000 collected patients from divers' study sides (Calw, Tübingen, Sindelfingen, Bayreuth) admitted to their hospitals suspected for coronary artery disease or severe aortic valve stenosis between 2012 and 2017 (with known age, gender). Each patient gave signed consent for the anonymous collection of all clinical data. The local ethical committee approved the study. Non-invasive cardiac CT examination and analysis were assessed in a blinded fashion to define individually the status of the coronary arteries and to describe the aortic valve area. Three hundred $(n=300)$ patients of the 1000 included patients in this study had given electrocardiography as well as weight and height to assess body mass index. The final assessment of the study data was performed in Calw, all non-invasive cardiac multi-sliced computed tomography scans were performed in all involved study sides (multicenter). In Calw, a team of very experienced cardiologists $(n=3)$ assessed finally together the data of correct angles of left ventriculography. Single observer differences were therefor ruled out. Experience is maintained and established through the German Society of Cardiology and certificated for all cardiac observers.

\section{Cardiac CT}

All scans were performed using the Philips Brilliance 256 MDCT iCT system $(0,6 \mathrm{mmx} 256$ collimation) (Philips, Amsterdam, The Netherlands). The patient's heart rate was optimized (50-60/min) with administration of metoprolol succinate $[4,5]$. Contrast enhanced scans were performed during breath-holding, with an intravenous infusion
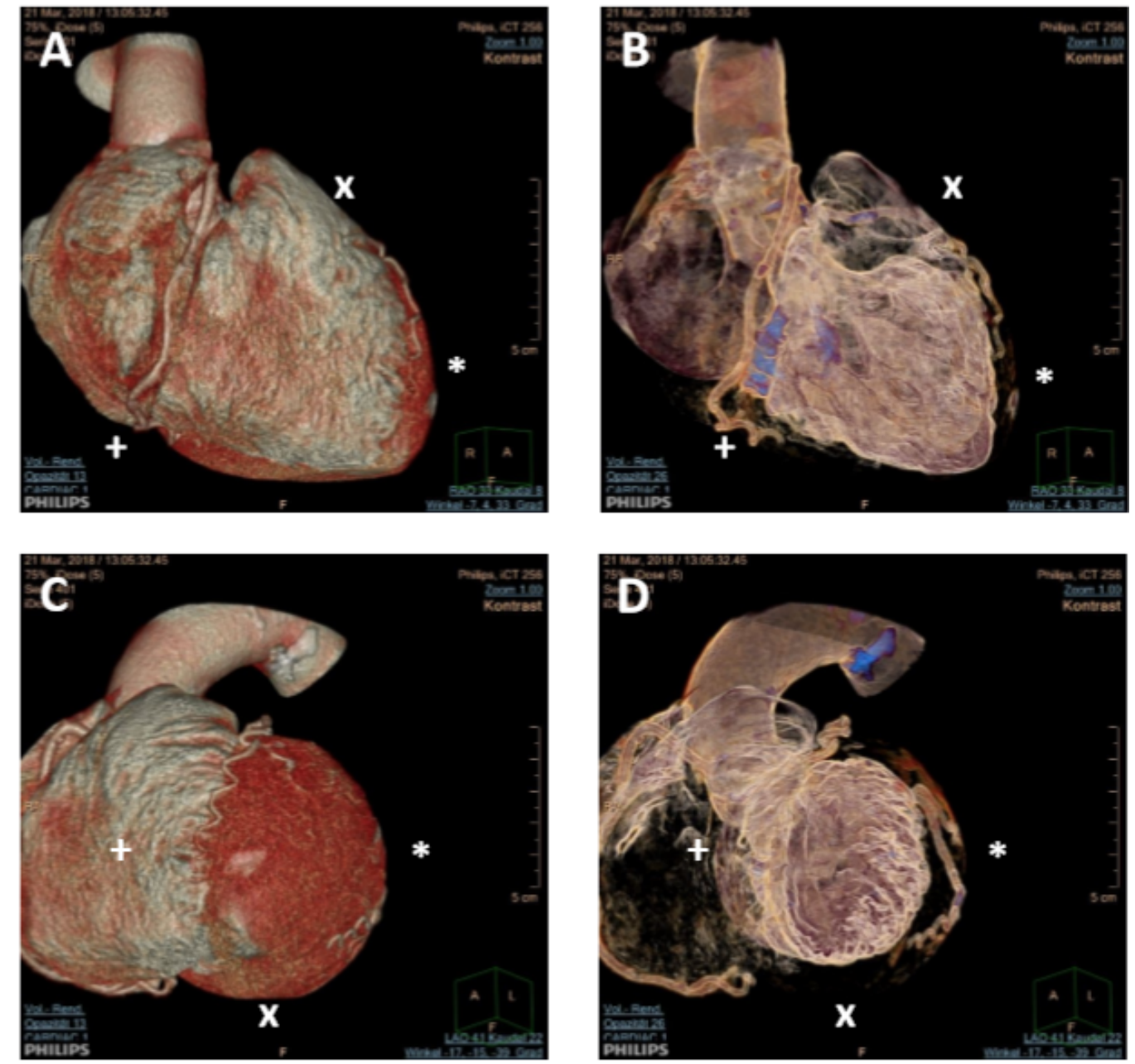

Figure 1. 3D Scans of Cardiac 256 Multi-Sliced Computed Tomography. Demonstrated here as example cardiac CT 3D scans from one female 77years old patient angulated to see inferior wall $(+)$, apical wall $(*)$ or anterior wall $(\mathrm{x})$ as seen by using RAO $30^{\circ}$ Caudal RAO $0^{\circ}$ performing invasive left ventriculography (panel A and B) as well as septal wall $(+)$, posterior wall $(\mathrm{x})$ and lateral wall $\left(^{*}\right)$ as seen by using LAO $60^{\circ}$ / Caudal LAO $0^{\circ}$ (panel C and D). In regard to generally used angulations performing left ventriculography, this patient will have uncorrect used angulations to describe regional wall motion stress and therefore catheter-based angulations should have been changed dramatically 
of $80 \mathrm{ml}$ contrast medium (Iomeprol $350 \mathrm{mgI} / \mathrm{ml}$ - Imeron 350, Bracco Imaging Deutschland $\mathrm{GmbH}$, Germany), using a tracking bolus system to commence scanning. A cardiac helical protocol or a step and shoot protocol using $\mathrm{x}$-ray reduction on male/ female patients respective were used to establish retrospective data, in $0.6 \mathrm{~mm}$ slice thickness, in order to create different reconstructions: a $65 \% \mathrm{RR}$ interval regardless of heart rhythm (sinus rhythm or atrial fibrillation) was chosen and a 3D model was reconstructed to align individually to the anterior/ apical and inferior wall (in comparison to left ventriculography: RAO $30^{\circ}$ ) and to posterior/ septal as well as lateral wall (in comparison to left ventriculography: $\mathrm{LAO} 60^{\circ}$ ). The chosen visual angulation parameters were given through the reconstructed 3D model (Philips, Amsterdam, The Netherlands) and marked individually for all patients through the team of experienced user observation (experienced invasive cardiologists).

Cardiac hypertrophy was defined as septal wall thickness above $11 \mathrm{~mm}$ in diastole and was marked as well.

\section{Statistical analysis}

Data represent mean \pm Standard Error of the Mean (SEM) as indicated. Statistical differences to characterize the mean angulation in LAO/Caudal_LAO/RAO/Caudal_RAO [in ${ }^{\circ}$ ] of different groups were assessed through unpaired t-test using correction of Bonferroni. To test for variance between the groups, a multivariate analysis of covariance with angulation as dependent variables, body mass index, age, hypertrophy as covariates and gender as fixed factor was performed. To define predictors for described angulations, we assessed multiple linear regression analyses [Durbin-Watson's equation]. To demonstrate correlation of miscellaneous variables to angulation we performed statistical correlation analyses [Pearsons' analysis]. All statistical tests were performed with SPSS Statistics software, version 25.0 (IBM/SPSS, Inc., Chicago, IL, USA). A p value $<0.05$ was considered statistically significant and marked directly in the graphs and tables.

\section{Results}

\section{Epidemic data}

We enrolled in total $n=1000$ collected patients presenting for 256 multi-sliced cardiac computed tomography on different study sides (Figure 1) with mean age of $63,91 \pm 13,73$ years [mean \pm SEM]. Forty percent (40\%) of the patients were female, $60,3 \%$ showed left ventricle hypertrophy (LV-hypertrophy) and the average body mass index (BMI) was $27,67 \pm 0,38$. From $n=300$ patients we collected body mass index and performed electrocardiography (ECG).

\section{Grouping analyses of described parameters}

We analyzed different patient dependent parameters (age, LVhypertrophy, BMI, QRS axis of the electrocardiography (ECG) and gender), grouped them respectively to clinical issues and performed student's t-test using Bonferroni correction for significance analyses (Figure 2). The grouping was done for body mass index (below 25, between 25 and 30, or above 30), left ventricular hypertrophy (Nonor existing LV-hypertrophy), gender (male or female), age (below 50 , between 50 and 70 , or above 70 years), and QRS axis of the ECG (vertical axis [VA], intermediate axis [IA], left axis deviation [LAD], marked left axis deviation [MLAD]. In general, all patient data shows different angulations for $\mathrm{LAO} 45^{\circ} \pm 8,70^{\circ}$,

Caudal_LAO $17^{\circ} \pm 6,95^{\circ}$, RAO $32^{\circ} \pm 8,63^{\circ}$, and Caudal_RAO $6^{\circ}$ $\pm 3,85^{\circ}$, respectively. Focusing for $\mathrm{LAO}$ (Figure 2 and $\mathrm{LAO}^{\circ}$-column), intended, we found statistical significant differences in age [above 70 years old: $47^{\circ} \pm 9,24^{\circ}$, gender [male: $42^{\circ} \pm 8,73^{\circ}$ ], and QRS axis of the ECG [marked left axis deviation: $50^{\circ} \pm 14,95^{\circ}$ ] - but none for BMI [above 30: $45^{\circ} \pm 8,7^{\circ}$ ], and LV hypertrophy [non: $46^{\circ} \pm 8,97^{\circ}$ ].

\section{ECG as parameter to predict correct angulations performing left ventriculography}

We analyzed 300 collected patients with subsequent 12-lead ECG and assessed the amplitude of I, V2 and aVF (negative [in $\mathrm{mV}]$ ) as predictive $3 \mathrm{D}$ model describing main vector of electric current flow as correction for angulations ideally prior to perform an invasive left ventriculography. Unfortunately, predicted through the literature, ECG parameters others then the QRS axis of the ECG are not eligible to describe predictive parameters to assume correct angulation. Seen the "invalid" data, we stopped following these parameter interpretation (data not shown).

\section{Group variance tests and predictors for correct angulation}

To test statistically whether there are differences within the groups or within the different groups, we tested statistical significance using (i) the analysis for multivariate variance and (ii) linear regression to define independent predictors for the different angulations. We saw different groups as independent predictors for the changed angulations to perform invasive left ventriculography as indicated bold. I.e. independent parameters for statistically significant changed angulations other than LAO $60^{\circ} /$ Caudal_LAO $0^{\circ}$ or RAO $30^{\circ} /$ Caudal_RAO $0^{\circ}$ as generally used are the age, the BMI, the gender, and some QRS axis of the ECG - highlighted bold.

\section{Correlations of independent parameters changing angulations}

Focusing on these previously described independent predictors of the changed angulations for the invasive left ventriculography in respect to regional wall motion stress we analyzed for statistical correlations using Pearson's equation and we found (Figure 3) correlations to age, to gender, to BMI, to LVhypertrophy, and to the QRS axis of the ECG describing the correct angulation. Age correlates statistically significant with the changed angulations for all used angulations. The other parameters are involved in some of the angulations. Visualization of the correlation lead us to (Figure 3 ) - indicating here as example the age as independent parameter to describe different angulations over the time.

\section{Recipes for chosen angulation angles to perform left ventriculography}

All collected data summarize a recipe to perform invasive left ventriculography in age-, BMI-, QRS axis of the ECG and gender dependent manner. Thereby, the main impact comes through the parameter age changing the LAO angle from $60^{\circ}$ to divers: older patients need LAO angle tipped to $50^{\circ}$, younger patients flatter to $42^{\circ}$. Male patients change the Caudal_LAO angle more to $20^{\circ}$, whereas female held the Caudal_LAO angle to $16^{\circ}$. LV-hypertrophy does not impact the clinical practice at all. ECG failed instead for the changed angulations. The QRS axis of the ECG previously performed prior to invasive left ventriculography changes only the patient dependent angulation parameters for all used angulations when marked left axis deviation is seen: LAO $50^{\circ}$ /Caudal_LAO $15^{\circ}$, RAO $20^{\circ}$ /Caudal_RAO $5^{\circ}$. LV-hypertrophy seems to change Caudal_LAO more to $18^{\circ}$, BMI over 30 reduces Caudal_LAO to $11^{\circ}$. RAO seems to be unchanged throughout the different groups. Caudal_RAO in general seems to be clearly favorite for $4^{\circ}$ when $\mathrm{BMI}$ is more than 30 . 
A

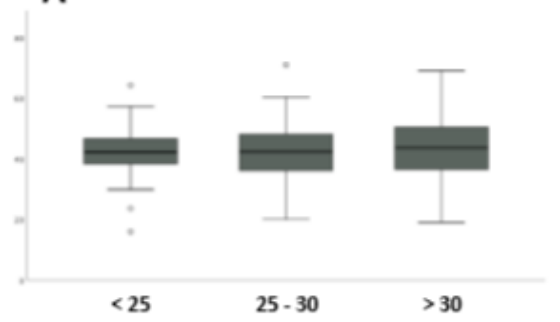

C

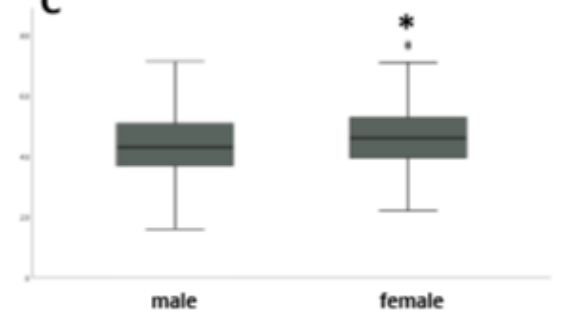

B

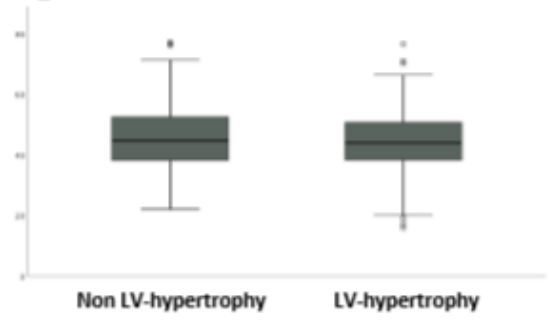

D

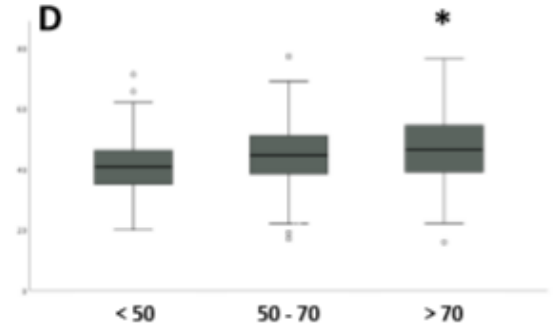

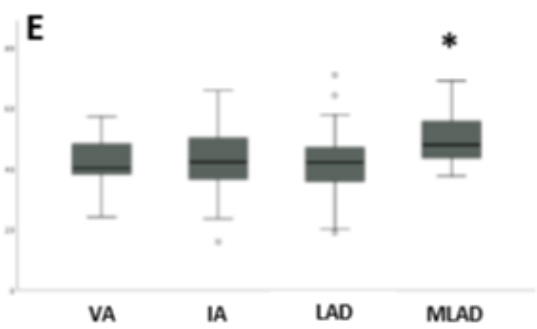

Figure 2. Group-Analyses of Significance for Correct LAO $60^{\circ}$ Angulations. Demonstrated here as example the grouped data of all enrolled patients assessed for correct LAO angulations in regard to the left ventriculography performed for regional wall motion stress (posterior, septal and lateral) describing statistical significant differences in the grouped parameters: body mass index (below 25, between 25 and 30, or above 30 - see panel A), left ventricular hypertrophy (Non- or existing LVhypertrophy - see panel B), gender (male or female - see panel C), age (below 50, between 50 and 70, or above 70 years - see panel D), and QRS axis of the ECG (vertical axis [VA], intermediate axis [IA], left axis deviation [LAD], marked left axis deviation $[\mathrm{MLAD}]$ - see panel E), as indicated respectively. A p-value below 0.016 (Bonferroni correction used) is mentioned statistically significant and revealed differences to the other groups within the same parameter as highlight by $* .{ }^{\circ}$ marks for statistic outlier data
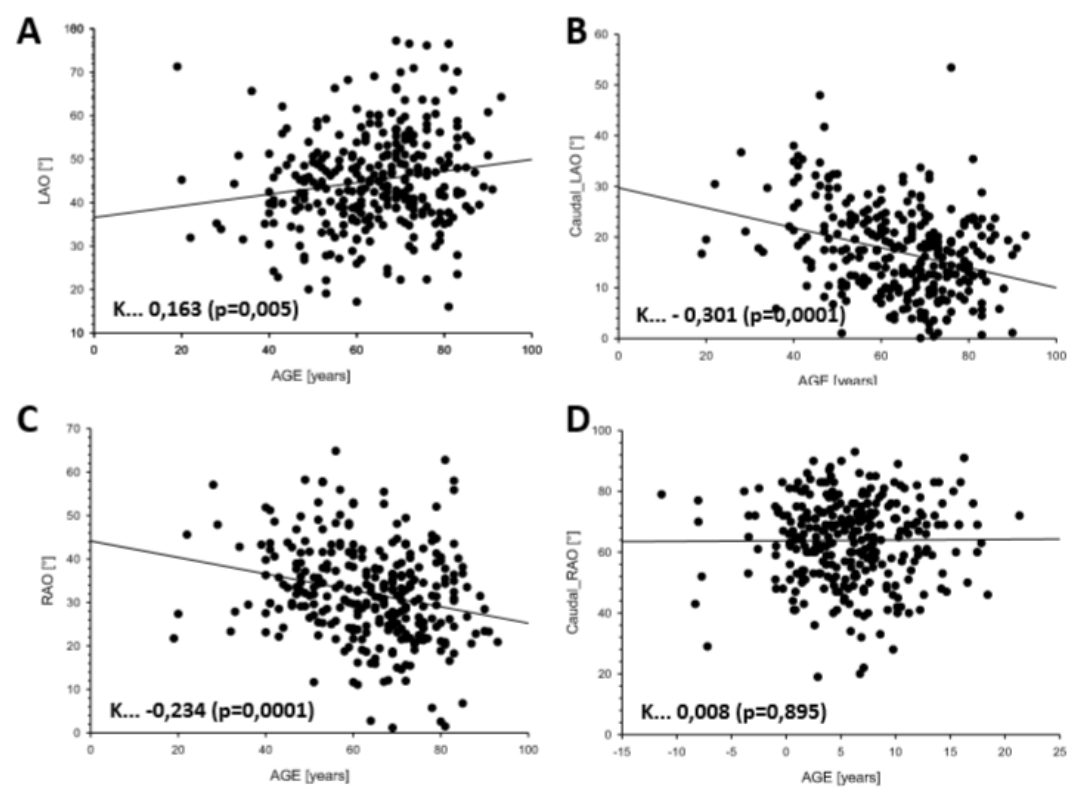

Figure 3. Correlations for all ideally correct Angulations with the Age [years]. Shown here as example for the parameter "Age [in years]" the correlation analyses (Pearson's equation) for all assessed angulations in correct and original matters while performing ideally left ventriculography. Statistical relevant Age dependent correlations of indicated angulations are marked through the coefficient $\mathrm{K}$ and highlighted significant through the $\mathrm{p}$-value in round brackets. A positive $\mathrm{K}$ value indicates increasing angulation parameters with the age, a negative $\mathrm{K}$ value stands for decreasing angulation parameters while growing up. LAO is demonstrated in panel A, Caudal_LAO in panel B, RAO in panel C, and Caudal_RAO in panel D, as indicated. Caudal_RAO does not correlate to the age (panel D) 


\section{Discussion}

These are retrospective multi-center $n=1000 / 300$ data from noninvasive cardiac 256 multi-sliced computed tomography scans analyzed for correct angulations to ideally perform left ventriculography (Figure $1)$.

In general, cardiac magnetic resonance imaging (MRI) is the commonly used technique for the assessment of left ventricular function [6]. Additionally, non-invasive cardiac CT is also established to characterize left ventricular function in terms of global strain [7] or reginal wall motion stress $[3,8]$ as well as to increase transcatheter aortic valve implantation [9-13] and to simplify stent positioning on severe coronary artery disease [14].

Our data is sampled for LV-hypertrophy, body mass index (BMI), gender (male/female), electrocardiography (ECG), age and QRS axis of the ECG. We analyzed all statistical variants and figured out significant predictors of left ventriculography dependent angulations as age, LV hypertrophy, gender, BMI and QRS axis of the ECG. The ECG itself as parameter for the electric main vector for alignment does not reflect any impact as predictor for miscellaneous angulations (data not shown). All others, mainly the age reflect as predictor for different angulations performing correct invasive left ventriculography (Figure 3 ). We set a recipe to perform an invasive left ventriculography corrected by age, BMI, gender, LV hypertrophy and QRS axis of the ECG. All examined parameters influenced the angulations statistically significant - except the single ECG for each individual patient.

The characteristic of the study (retrospective, multi-center assay, single center assessment of the shown data) does not influence the outcome of the data. The experience of the CT users underlines the reflection of the printed data. The power of the data is clearly met $(n=1000 / n=300 / 1000)$, the statistical analysis is correct as well.

Thereby, we underline the correctness of the divers changes in angulations to perform an invasive left ventriculography in the cath-lab.

\section{Conclusion}

This retrospective multi-center study clearly emphasizes a change in the set of angulation angles performed in the invasive cath-lab for regional wall motion stress age-/BMI-/LV hypertrophy and genderdependent as demonstrated. We declare a recipe for performing invasive retrograde left ventriculography depending on age, LVhypertrophy, gender and BMI in the set-up of Cath labs in Departments of Cardiology. Through our data, invasive retrograde characterization of LV function in the cath-lab, invasive cardiologists are forced to follow the recipe of miscellaneous angulations.

\section{Acknowledgement}

We would like to thank all involved 1000 patients given written consent to participate in this retrospective study to characterize the individual LV function. No funding was involved. No patient right was harmed, no patient got disadvantage negotiating the participation in this retrospective study. We thank all participants to this study addressing the efforts they maintained additionally to their normal work.

\section{References}

1. Koster O, Boldt I, Thurn P (1980) Complications of selective retrograde laevocardiography. A survey of 2000 examinations (author's transl). Rofo 133: 608614. [Crossref]

2. Luig H, Bartella R, Carstens B, Domovitz S, Reuter R, et al. (1979) The determination of the left ventrical volume curve without background correction and its validation by direct intercomparison with the ejection fractions as determined by biplane laevocardiography (author's transl). Nuklearmedizin 18: 120-124.

3. Pflederer T, Ho KT, Anger T, Krahner R, Ropers D, et al. (2009) Assessment of regiona left ventricular function by dual source computed tomography: interobserver variability and validation to laevocardiography. Eur J Radiol 72: 85-91. [Crossref]

4. Pflederer T, Achenbach S (2010) Aortic valve stenosis: CT contributions to diagnosis and therapy. J Cardiovasc Comput Tomogr 4: 355-364. [Crossref]

5. Achenbach S, Delgado V, Hausleiter J, Schoenhagen P, Min JK, et al. (2012) SCCT expert consensus document on computed tomography imaging before transcatheter aortic valve implantation (TAVI)/transcatheter aortic valve replacement (TAVR). $J$ Cardiovasc Comput Tomogr 6: 366-380. [Crossref]

6. Benameur N, Caiani EG, Alessandrini M, Arous Y, Abdallah NB, et al. (2018) Left ventricular MRI wall motion assessment by monogenic signal amplitude image computation. Magn Reson Imaging 54:109-118. [Crossref]

7. Marwan M, Ammon F, Bittner D, Rother J, Mekkhala N, et al. (2018) CT-derived left ventricular global strain in aortic valve stenosis patients: A comparative analysis pre and post transcatheter aortic valve implantation. J Cardiovasc Comput Tomogr 12: 240244. [Crossref]

8. Pourmorteza A, Keller N, Chen R, Lardo A, Halperin H, et al. (2018) Precision of regional wall motion estimates from ultra-low-dose cardiac CT using SQUEEZ. Int $J$ Cardiovasc Imaging 34: 1277-1286. [Crossref]

9. Hell MM, Biburger L, Marwan M, Schuhbaeck A, Achenbach S, et al. (2017) Prediction of fluoroscopic angulations for transcatheter aortic valve implantation by CT angiography: influence on procedural parameters. Eur Heart J Cardiovasc Imaging 18: 906-914. [Crossref]

10. Schuhbaeck A, Weingartner C, Arnold M, Schmid J, Pflederer T, et al. (2015) Aortic annulus eccentricity before and after transcatheter aortic valve implantation: Comparison of balloon-expandable and self-expanding prostheses. Eur J Radiol 84: 1242-1248. [Crossref]

11. Schmidkonz C, Marwan M, Klinghammer L, Mitschke M, Schuhbaeck A, et al (2014) Interobserver variability of CT angiography for evaluation of aortic annulus dimensions prior to transcatheter aortic valve implantation (TAVI). Eur J Radiol 83: 1672-1678. [Crossref]

12. Arnold M, Achenbach S, Pfeiffer I, Ensminger S, Marwan M, et al. (2012) A method to determine suitable fluoroscopic projections for transcatheter aortic valve implantation by computed tomography. J Cardiovasc Comput Tomogr 6: 422-428. [Crossref]

13. Wuest W, Anders K, Schuhbaeck A, May MS, Gauss S, et al. (2012) Dual source multidetector CT-angiography before Transcatheter Aortic Valve Implantation (TAVI) using a high-pitch spiral acquisition mode. Eur Radiol 22: 51-58. [Crossref]

14. Schlundt C, Kreft JG, Fuchs F, Achenbach S, Daniel WG, et al. (2006) Threedimensional on-line reconstruction of coronary bifurcated lesions to optimize sidebranch stenting. Catheter Cardiovasc Interv 68: 249-253. [Crossref]

Copyright: (C2019 Anger T. This is an open-access article distributed under the terms of the Creative Commons Attribution License, which permits unrestricted use, distribution, and reproduction in any medium, provided the original author and source are credited. 